\title{
Scheitert die planerische Umsetzung von Gegebenheiten des Naturhaushaltes an den vorhandenen gesetzlichen Mitteln?
}

\section{Einleitung}

Naturhaushalt und Planung - ein Spannungsfeld größter Brisanz, eine Problematik mit Diskussionen ohne Ende.

Die Umsetzung der Erkenntnisse über den Naturhaushalt in der Planung erweist sich als äußerst schwierig, auch wenn wir die Diskussionen der Beweisführung auf der Seite lassen.

\section{Warum eigentlich?}

Stehen uns nicht ausgezeichnete gesetzliche Mittel zur Verfügung? Haben wir nicht ein Raumplanungsgesetz, in dem der Schutz der natürlichen Lebensgrundlagen gefordert wird? Haben wir nicht ein äuBerst restriktives Forstgesetz? Umweltschutzgesetz, Naturschutzgesetzgebungen, Gewässerschutzgesetz...

Der Titel dieser Ausführungen ist angesichts der Gesetzesfülle zu Faktoren des Naturhaushaltes reinste Provokation. Durch Provokationen werden Denkund Arbeitsweisen angezweifelt, werden hinter gültigen Normen Fragezeichen gesetzt. Durch diese Fragezeichen ist es möglich, grundsätzliche Fehler zu erkennen und vielleicht neue Lösungsansätze zu entwickeln. In einer Zeit, in der jede Aussage von Experten/-innen zuerst einmal angezweifelt wird, ist eine dauernde und grundsätzliche Auseinandersetzung besonders wichtig, hängt doch unsere Glaubwürdigkeit davon ab.

\section{See- und Flußufergesetz im Kanton Bern}

Das See- und Flußufergesetz im Kanton Bern (SFG) vom 6. Juni 1982 ist etwas eigenartiges: nicht nur weil es durch eine Initiative - trotz Gegenvorschlag - zustande gekommen ist, sondern weil es so scheinbar widersprüchliche Nutzungsansprüche wie Erholung, Baubeschränkungen und Erhaltung von Naturlandschaften als Inhalt hat:

Art. 3 Der Uferschutzplan legt namentlich fest:

a eine Uferschutzzone im unüberbauten Gebiet und Baubeschränkungen im überbauten Gebiet

b einen Uferweg c allgemein benutzbare Freiflächen für Erholung und Sport

d Maßnahmen zur Erhaltung naturnaher Uferlandschaften und zu ihrer Wiederherstellung

Zusätzlich werden im Gesetz das Verfahren, die Zuständigkeit, die Realisierung und die Finanzierung in einer ungewohnt detaillierten Weise festgelegt.

Im Moment bearbeiten die betroffenen Gemeinden die Uferschutzpläne, welche die Maßnahmen grundeigentümerverbindlich festlegen. Die planerische Umsetzung ist also in vollem Gang.

Ein besonders interessanter Aspekt zum Thema «Naturhaushalt und Planung» ist in Artikel 3, Absatz d enthalten:

Maßnahmen zur Erhaltung naturnaher Uferlandschaften und zu ihrer Wiederherstellung.

Den Initianten und der stimmenden Bevölkerung dürfte die planerische Tragweite dieses Artikels wohl kaum voll bewußt gewesen sein. Es wird nicht von Ufern, sondern von Uferlandschaften gesprochen, und die Wiederherstellung einer naturnahen Landschaft dürfte eines der schwierigsten planerischen Probleme überhaupt sein. In den Richtlinien SFG (RPA, 1986) werden natürliche und naturnahe Uferlandschaften definiert:

Natürliche Uferlandschaften enthalten eine vollständige, den gegebenen Verhältnissen (Geländeform, Klima, hydraulische Verhältnisse) angepaßte natürliche Abfolge der Pflanzen vom Wasser zum Land.

Naturnahe Ufer(landschaften) enthalten Teile dieser Abfolge.

Aus dieser Definition geht hervor, daß nicht jede Bepflanzung als naturnah bezeichnet werden darf. Jedes Ufer muß demnach auf seine «Natürlichkeit» hin überprüft werden. Zusammen mit den übrigen Ansprüchen an die See- und Flußufer wird nun entschieden, wo welche Maßnahmen zur Erhaltung und Wiederherstellung naturnaher Ufer durchzuführen sind. Anscheinend bereitet die Umsetzung des Art. 3.d in den Uferschutzplänen einigen Ge-

Peter Hirsig, Dr. phil.; Jürg Petermann, dipl. Geograph, «naturaquan, Thunstr. 101a, 3006 Bern 
meinden mit ihren Ortsplanern ausgesprochen Mühe. Beim Kanton sind Pläne zur Vorprüfung eingegangen, in welchen dieser Artikel 3.d einfach ignoriert wurde.

\section{Fallbeispiel «Aarelandschaft Thun-Bern»}

In der "Aarelandschaft Thun-Bern" ist die Umsetzung dieses Gesetzesartikels besonders interessant. Infolge der ausgedehnten Auenlandschaften handelt es sich um ein Naturschutzgebiet von nationaler Bedeutung (BLN-Objekt Nr. 1314). Wegen der Bedeutung des Gebietes hat der Kanton beschlossen, den Gemeinden nach einheitlichen Kriterien erarbeitete Grundlagen bezüglich Erhaltung und Wiederherstellung zur Verfügung zu stellen.

Warum wurde gerade ein Naturschutzgebiet als Fallbeispiel für die planerische Umsetzung von Maßnahmen zur Erhaltung von naturnahen Uferlandschaften ausgewählt? Hier ist doch die Planung kein Problem, da die Anliegen der Natur im Vordergrund stehen. Aber zwischen Thun und Bern macht nicht nur der Naturschutz seine Ansprüche geltend, sondern auch andere Nutzungen wollen in diesem Gebiet berücksichtigt werden:

- Die Auenlandschaft als Trinkwasserfassungsgebiet von zumindest regionaler Bedeutung.

- Gewässerregulierung/Wassernutzungen im Oberund Unterlauf haben Auswirkungen auf das Wasserregime im Gebiet.

- (Intensive) Forst- und Landwirtschaft
- Kiesbau

- Autobahn

- Erholung

Und nun wird im See- und Flußufergesetz die Erhaltung und Wiederherstellung naturnaher Uferlandschaften gefordert. Das heißt u. a., ein Mindestmaß an landschaftlicher Dynamik muß gewährleistet, in einer Flußauenlandschaft sollten also teilweise wieder Überflutungen möglich sein.

Sind die Beziehungen der verschiedenen Nutzungsarten in einem solchen Gebiet nicht vollständig geregelt - sicher auch eine Aufgabe der Planung -, wird die praktische, grundeigentümerverbindliche Umsetzung eines solchen Artikels entweder ein Ding der Unmöglichkeit oder der Inhalt des Artikels wird völlig ausgehöhlt.

Wie ist das zu verstehen? Jede wirkliche Wiederherstellungsmaßnahme - und dabei sprechen wir nicht von Begrünungsmaßnahmen der Uferböschung jede solche Maßnahme tangiert eine große Anzahl von verschiedenen Gesetzen, welche die Anliegen der einzelnen Nutzungen regeln. $\mathrm{Zu}$ jedem Nutzungsanspruch kann weiter eine Fachstelle zugeordnet werden, welche diese Interessen verwaltet, kontrolliert und schützt. Anfallende Probleme werden von den Fachstellen, den Gemeinden und beigezogenen Fachleuten meist nach fachspezifischen, isolierten Lösungsansätzen bearbeitet. Dieses Vorgehen ergibt bei einer Aufgabe, in der verschiedene Nutzungsarten starke Ansprüche an das Gebiet erheben, schon auf kantonaler Stufe grundsätzliche

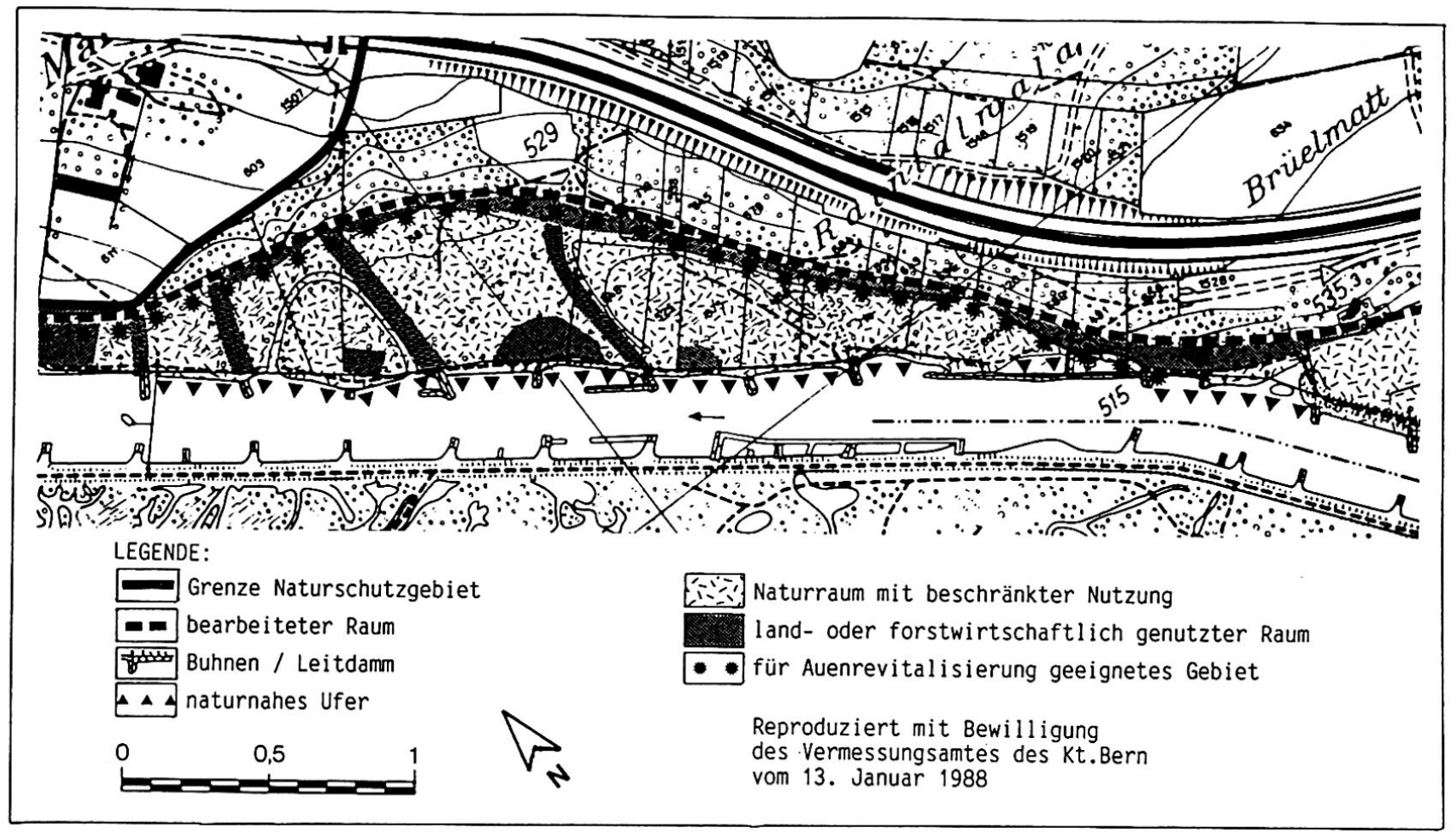

Abb. 1 Situation in einem ausgewählten Abschnitt an der Aare zwischen Thun und Bern 
Schwierigkeiten, bevor die eigentliche planerische Umsetzung beginnen kann.

In unserem Fallbeispiel haben wir folgende Situation:

- Die Erhaltungs- und Wiederherstellungsmaßnahmen der naturnahen Ufer sind direkt abhängig von der Nutzung im dahinterliegenden Gebiet. Eine überflutete Aue fordert andere Maßnahmen an der Uferböschung als beispielsweise ein Trinkwasserfassungsgebiet.

- Die einzelnen Teilräume stehen in einem direkten Zusammenhang mit dem ober- und unterliegenden Gebiet.

- Maßnahmen am Ufer können das Wasserregime der Aare beeinflussen und haben deshalb Auswirkungen auf das ganze Flußsystem.

Ohne Festsetzung der Nutzungsziele in den einzelnen Teilräumen und im gesamten Gebiet zwischen Thun und Bern sowie ohne Überprüfung allfälliger Auswirkungen auf Ober- und Unterlieger ist eine Wiederherstellung von naturnahen Uferlandschaften nicht möglich, ja sie muß von den einzelnen zuständigen Stellen aus grundsätzlichen Überlegungen (fehlende Entscheidungsgrundlagen) abgelehnt werden.

Im ausgewählten Uferabschnitt (Abbildung 1) wird im heutigen Zustand die Überflutung der Uferlandschaft durch einen Leitdamm verhindert. Eine echte Wiederherstellungsmaßnahme bedeutet hier z. B. eine Revitalisierung der Flußaue. Diese hat aber Auswirkungen auf das Wasserregime und somit Ober- und Unterlieger. Die Veränderungen in der Regulierung der Aare zwischen Brienzersee, Bielersee und Murgenthal sind ohne eingehende Abklärungen nicht abzuschätzen, und das Wasserwirtschaftsamt muß aus hochwasserschutztechnischen Gründen die Maßnahme ablehnen.

Die Festsetzung der Nutzung bedingt konzeptionelle Arbeiten, in denen die einzelnen Fachbereiche ihre Ansprüche als Grundlage für eine Gesamtplanung geltend machen. Gerade in diesem Punkt finden wir eine der großen Schwachstellen der Planung. Zwar sind in verschiedenen Gesetzen allgemeine konzeptionelle Grundsätze - z. B.: der Artikel 1 des RPG - enthalten, aber die konkrete Planung wird im allgemeinen immer noch einzelsprungweise und sehr pragmatisch durchgeführt.

Zwischen Thun und Bern hat ein Bundesgerichtsentscheid im vergangenen Jahr auch wieder einmal deutlich aufgezeigt, zu welchen Ergebnissen diese Arbeitsweise führen kann. Es handelt sich um die Rodungsbewilligung Auwald Belp. Verschiedene Gemeinden in der Region Bern haben in ihrem Trinkwasser Nitratgehalte, welche nicht der Lebensmittelverordnung entsprechen. Als Problemlösung wird nicht die Sanierung der gemeindeeigenen
Grundwasserkörper in Angriff genommen, sondern ein Ausmischen mit gutem Wasser geplant. Was liegt hier näher, als neue Brunnen in einer Auenlandschaft? Diese Brunnen haben eine Absenkung des Grundwasserspiegels und damit die Zerstörung von einigen Hektaren Auenwald zur Folge. Die Rodungsbewilligung zum Bau dieser Anlagen wurde nun vom Bundesgericht erteilt.

Hier wird deutlich, wo die Planung und somit auch die Umsetzung der Anforderungen zum Schutz unserer natürlichen Lebensgrundlagen krankt. Grundsätzliche planerische Fragen - und das heißt schlußendlich politische Entscheide - werden immer weniger von Planern/-innen, der Verwaltung, Menschen aus Wissenschaft und Technik oder politischen Gremien gelöst, sondern den Gerichten zugehalten. Dadurch können wir uns sehr elegant der politischen und wissenschaftlichen Verantwortung entziehen. Mit diesen heute praktizierten Ansätzen ist die Umsetzung des Artikels 3, Abs. d des SFG nicht nur zwischen Thun und Bern von vorneherein zum Scheitern verurteilt; er wird zum reinen «Begrünungsartikel» deklassiert.

\section{Schlußgedanken}

Die Antwort auf die Frage im Titel - Scheitert die planerische Umsetzung von Gegebenheiten des $\mathrm{Na}$ turhaushaltes an den vorhandenen gesetzlichen Mitteln? - kann nicht eindeutig gegeben werden. Der Grund zum Scheitern liegt tiefer. Die Gesetze sind nur der Ausdruck unserer Lösungsansätze und Arbeitsweisen, mit denen wir die Probleme angehen. Die Umsetzung scheitert vielmehr an fehlenden Konzepten und unklaren Zielvorstellungen.

\section{Kann diese Situation überhaupt verbessert werden?}

Der Beantwortung dieser Frage muß eigentlich ein ganzes Seminar gewidmet werden, bzw. eine permanente Diskussion darüber stattfinden. Trotzdem seien hier einige Gedanken summarisch dargestellt.

\section{Politische Verantwortung:}

Die politische Verantwortung sowohl von Einzelnen wie auch von Gruppen darf nicht der Rechtssprechung übertragen werden. Gerade WissenschafterInnen müssen ihre politische Verantwortung wahrnehmen und dürfen sich nicht hinter einer sogenannten Objektivität verstecken.

\section{Zielvorstellungen und Konzepte:}

Gerade Natur- und Landschaftsschutz müssen endlich die konventionelle Arbeitsweise - d. h. Einzelobjektschutz - über Bord werfen und konzeptionell arbeiten. 


\section{Verfassung und Gesetze:}

Die Verfassung und die Gesetze müssen ausgemistet werden. Der Schutz unserer Lebensgrundlagen und somit unserer Existenz muß in den Vordergrund gerückt werden. "UVP» von Verfassung und Gesetzen.

\section{Verwaltungsstruktur}

Alle Probleme dürfen nicht mehr fachbereichsweise, sondern in einem räumlichen und zeitlichen Gesamtzusammenhang betrachtet und gelöst werden. Es ist klar, daß dadurch jede historisch gewachsene Verwaltungsstruktur verändert werden muß.

An einer Tagung der Gesellschaft für angewandte Geographie sind viele dieser Gedanken nichts Neues. Trotzdem oder vielleicht auch deshalb können wir es nicht unterlassen, zum Schluß auch noch auf das ach so viel diskutierte Bild der Geographen/ -innen einzutreten. Die Geographie als zusammen- führende Disziplin kann einen wichtigen Beitrag zur Erhaltung des Biotopes Mensch leisten. Nur müssen Denkweise und damit auch das Vermögen zu breit angelegten Lösungsansätzen entsprechend geschult werden. Es stimmt deshalb schon nachdenklich, wenn gerade an den Deutschschweizer Universitäten die Tendenz besteht, daß die Ausbildung zu Geotechnikern - Hydrologen, Geomorphologen, Klimatologen, Pedologen usw. - gefördert und die geographischen Ansätze immer mehr vernachlässigt werden. Verlieren wir als Geokraten nicht unsere einzige wirkliche Stärke?

\section{Literatur}

Raumplanungsamt des Kts. Bern (1986): Richtlinien SFG, Bern

Kanton Bern (1982): Gesetz über See- und Flußufer (SFG) 